\title{
Millimeter-Wave Integrated Side-Fire Leaky-Wave Antenna and its Application as a Spectrum Analyzer
}

\author{
Daniel J. King, Student Member, IEEE, Mohamed K. Emara, Student Member, IEEE, \\ and Shulabh Gupta, Senior Member, IEEE
}

\begin{abstract}
An analog, low-profile and shielded spectrum analyzer is proposed for operation at $\mathbf{m m}$-wave frequencies around the $60 \mathrm{GHz}$ band based on a novel side-fire Leaky-Wave Antenna (LWA) configuration. The proposed side-fire periodic LWA is systematically developed from a conventional 3-port waveguide T-junction which is modified to a LWA unit cell with an internal matching mechanism to suppress the stop-band and enable broadside radiation based on unit cell symmetry considerations. The resulting periodic side-fire antenna radiates in the plane of the antenna, whereby the leakage power be either be allowed to radiate in free-space or kept confined inside a PPW structure. The proposed side-fire structure thus can be completely shielded useful as an analog broadband spectrum analyzer using Substrate Integrated Waveguide (SIW) Technology. Furthermore, a convex side-fire antenna is demonstrated to focus the radiated beams in the near-field of the structure to make the entire system compact. The integrated spectrum analyzer is experimentally demonstrated between $59 \mathrm{GHz}$ - $66 \mathrm{GHz}$ providing $1 \mathrm{GHz}$ frequency resolution. Furthermore, a simple mathematical model consisting of array of line sources is proposed to efficiently model the beam-scanning characteristics of the curved side-fire LWA in the near-field of the structure.
\end{abstract}

Index Terms - Side-fire antennas, substrate integrated waveguides (SIW), spectrum analysis, Leaky-wave antennas (LWA), 5G systems, near-field focussing, conformal antennas, stop-band suppression, full-space beam-scanning.

\section{INTRODUCTION}

Spectrum analysis is among the most fundamental signal processing operations required in engineering and forms the basis of various systems in application areas such as signal distortion measurement from audio equipment, spectrum monitoring for governmental frequency allocation of various radio services, and electromagnetic emissions testing of equipment [1], [2]. Traditional techniques include discrete Fourier transforms (DFTs) based on digital computing for Radio Frequency (RF) signals, where an analog test signal is first sampled, discretized and then digitally processed to compute its Fourier transform [3], [4]. However, this approach suffers from performance degradation and challenging implementation as frequency increases and signal bandwidths become large [5]. With the upcoming next generation wireless systems, novel technologies for instrumentation and communication are required particularly in the IEEE $801.11 \mathrm{ad}(60 \mathrm{GHz}$ unlicensed band) mm-Wave band which are capable of handling large signal bandwidths with reduced latencies [6] [7].

D. J. King, M. K. Emara and S. Gupta are with the Department of Electronics, Carleton University, Ottawa, Ontario, Canada. (email: danielking3@cmail.carleton.ca)
Consequently, alternative techniques have been explored in the literature to perform spectrum analysis using analog methods. At optics, the material dispersion is utilized to realtime separate the various spectral components of a broadband test signal, commonly utilized in Real Time Fourier Transformers (RTFTs) [8], Arrayed Waveguide Gratings (AWG) [9], prisms or to use the wavelength dependent diffraction orders in periodic diffraction gratings [10] and Virtual Image Phased Arrays (VIPA) [11], for instance. While such systems are not as flexible as a digital system, they offer high acquisition bandwidth and ultrafast system operation. Inspired from these optical systems, various solutions have been proposed at RF using both guided and radiative systems based on dispersion (frequency dependent group delay, $\tau(\omega)$ ) engineered Bragg gratings [12], spatial interferometers [13] and leaky-wave Antennas (LWAs), for instance, exploiting their frequency scanning properties [14]-[18]. However, all of these solutions suffer from either lack of device integration or are based on unshielded microstrip implementations which are not particularly suitable for higher mm-wave bands (e.g. 60 $\mathrm{GHz}$, for instance) leading to significant free-space radiation, not ideal for minimal electromagnetic interference.

Recently, an idea of a novel mm-wave near-field real-time spectrum analyzer (RTSA) based on an integrated side-fire LWA has been proposed in [19]. Here this idea is further explored in details and experimentally demonstrated. The subsequent contributions of this works are two-fold: 1) It is based on a novel side-fire LWA using integrated waveguide periodic apertures on its side walls, providing a typical frequency scanned power leakage along the plane of the antenna as opposed to radiation normal to the antenna plane in conventional designs. The LWA unit cell is designed with a self-matching mechanism that suppresses the stop-band characteristics of periodic structures, thereby enabling seamless frequency scanning from the backward to forward region through broadside. 2) Its application to broadband spectrum analysis at mm-wave frequency bands. In this work, a detailed design analysis of a side-fire antenna and its application to spectrum analysis is presented along with full experimental demonstrations around the $60 \mathrm{GHz}$ band. The system is the first of its kind at mm-wave frequencies, and is fully integrated inside a low profile parallel-plate waveguide (PPW) with no radiation to free-space, eliminating any electromagnetic interference (EMI) with neighbouring instruments. The system is further designed to operate in the near-field region by introducing a curved shape of the structure resulting in compact size and lowprofile compatible with a standard printed circuit board (PCB) 

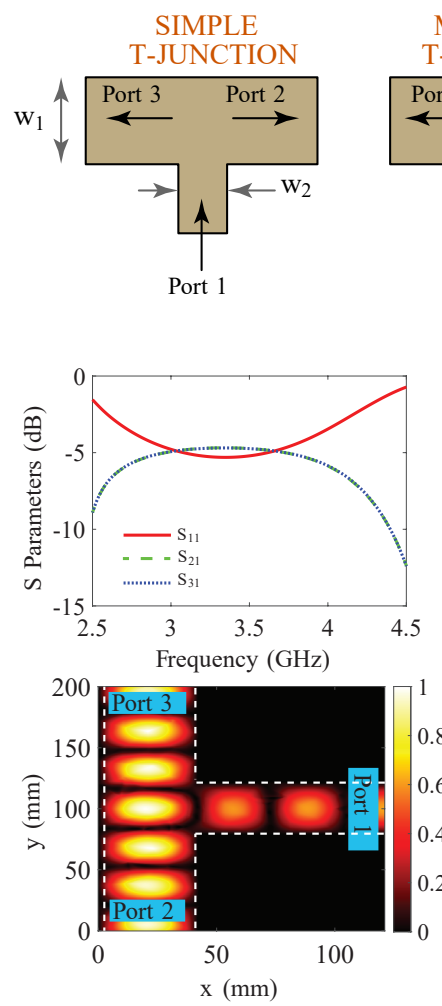

(b) Simple T-junction.

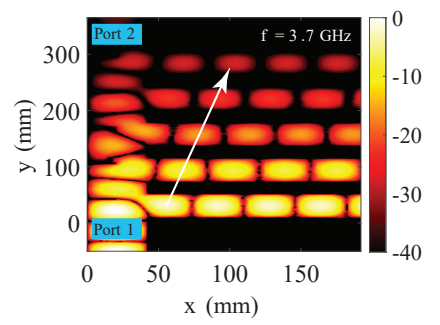

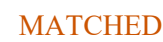

T-JUNCTION

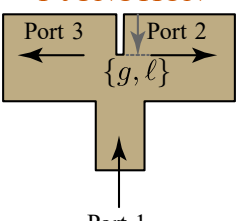

Port 1
PORT SWITCHED PORT SWITCHED SIMPLE T-JUNCTION MATCHED T-JUNCTION

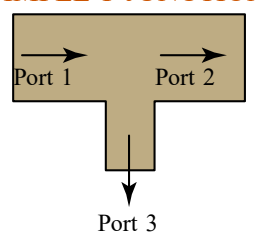

CASCADED MATCHED T-JUNCTION/SIDE-FIRE STRUCTURE

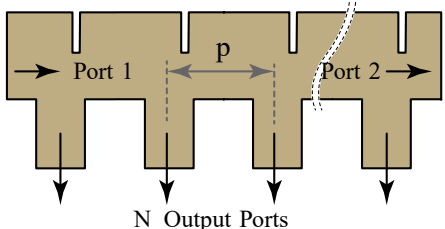

(a) Evolution of a conventional T-junction to an $N$-port power divider.
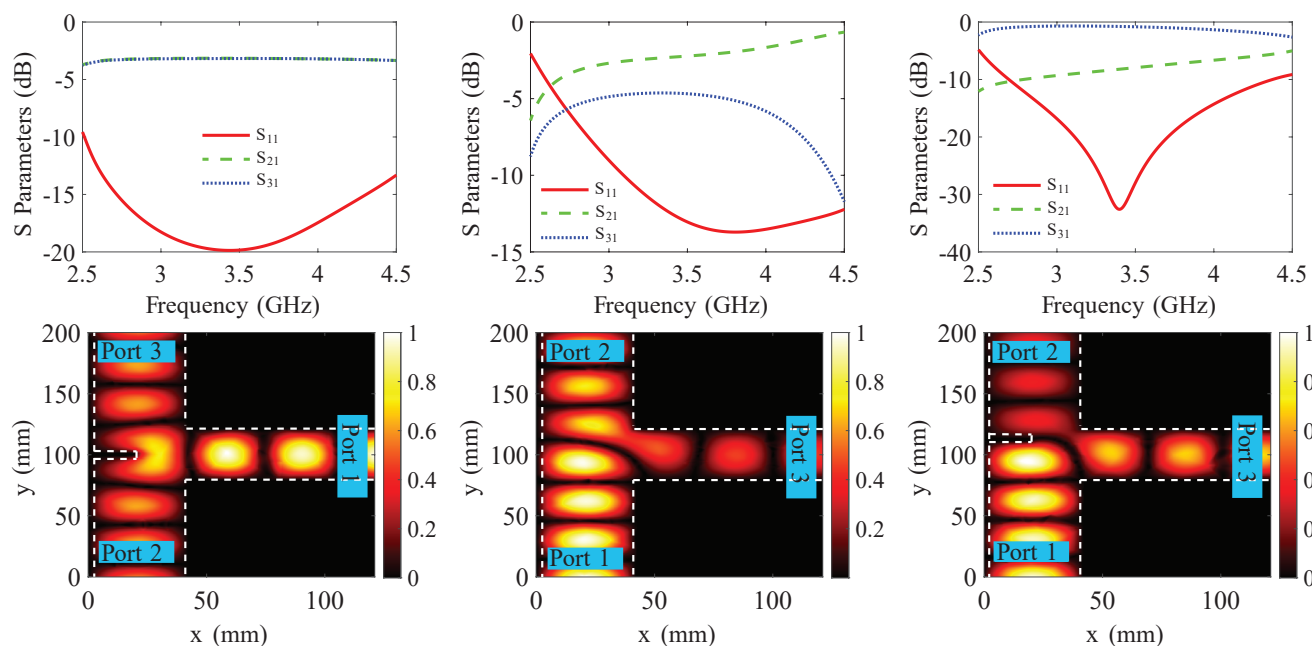

(c) Matched T-junction.
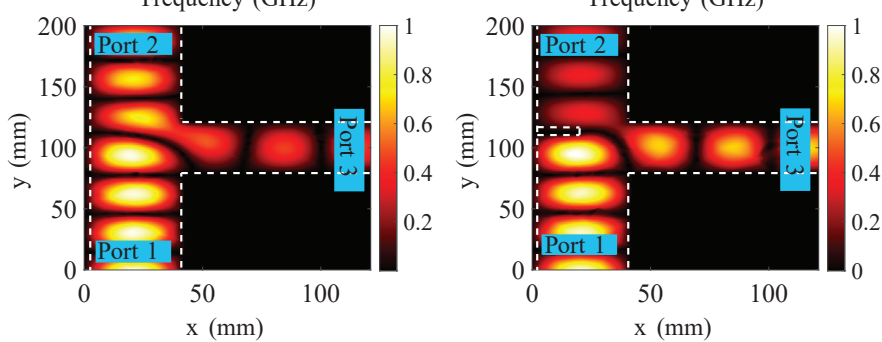

(d) Port switched simple T-junction.
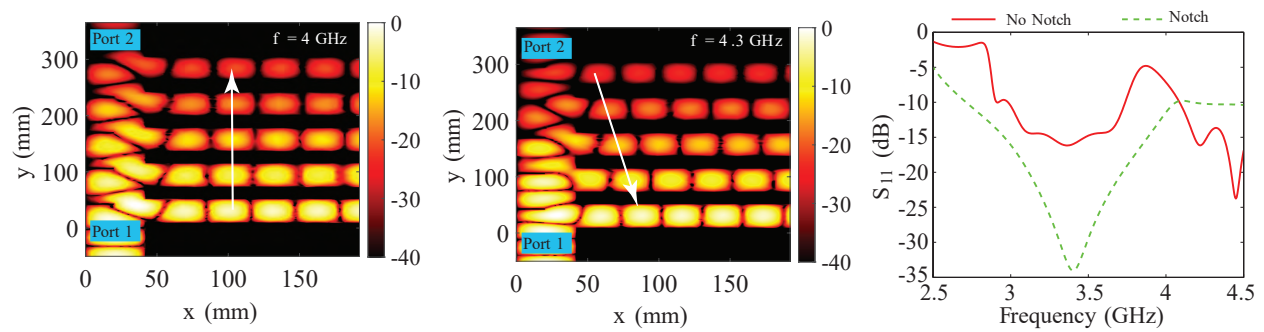

(f) Cascaded matched T-junction.

Fig. 1. Principle of constructing an $N$-port power divider starting from a conventional 3-port waveguide T-junction. Various design parameters are: $s=12$ $\mathrm{mm}, w_{1}=w_{2}=42 \mathrm{~mm}, p=63.32 \mathrm{~mm}$, notch length $\ell=20 \mathrm{~mm}$, and notch width $g=2 \mathrm{~mm}$. The fields are plotted at $f=4 \mathrm{GHz}$ for (b-e)

process.

This paper is organized as follows. Section II describes the principle of the proposed side-fire leaky-wave antenna inspired from a cascaded matched T-junction cell with an asymmetric notch (or inductive post in fabrication). Through cascading the T-junctions, the output ports can be opened for radiation forming a periodic LWA. Detailed eigenmode analysis is further provided to highlight important features of the structure. Section III shows how the side-fire LWA can be used as a real-time spectrum analyzer through the property of spatial-spectral decomposition. By introducing curvature, a compact size for the spectrum analyzer can be achieved. Furthermore, a simple analytical method is used to model the beam-scanning property of this structure providing further insight. Section IV provides experimental results showing the fabricated structures and the corresponding measurement results demonstrating the successful operation of the sidefire LWA for spectral decomposition. Finally, conclusions are provided in Sec. V.

\section{Proposed Side-Fire Leaky-Wave Antenna (LWA)}

A LWA is typically a 2-port traveling-wave structure that gradually radiates EM energy to free-space as the wave propagates along it [20]. It is characterized by a complex propagation constant $\gamma(\omega)=\alpha(\omega)+j \beta(\omega)$, where $\alpha$ is the leakage per unit length along the structure and $\beta$ is the phase constant. LWAs can be considered as series-fed phased arrays, and are typically broadband and directive in nature. While $\alpha$ (and the physical aperture) controls the beamwidth of radiation, $\beta$ provides the necessary frequency dependent phase shifts to scan the radiation beams. The Majority of LWAs are periodic in nature with near-sub-wavelength periodicities, where they radiate from the $n=-1$ space harmonics which fall within the fast-wave region with both backward and forward radiation [20]-[22]. On the other hand, uniform LWA structures based on metamaterial principles feature deep 
sub-wavelength periodicities and enable full-space frequency scanning using fundamental mode directly [23], [24].

The majority of LWAs provide frequency-scanned peak radiation normal (in the $y-z$ plane) to the plane of the antenna (say $x-y$ plane, with antenna along $y$-axis for instance). An exception is a rectangular waveguide based LWA, where a continuous slot is opened enabling peak radiation from the sides, in the $x-y$ plane [25]. Such a structure however is restricted to operate in the forward region only with no broadside radiation capability. Now, our objective here is to design a LWA, which is integrated into the subtrate, and radiates in the $x-y$ plane with radiation in both forward and backward regions including broadside. In this section, we will present a step by step procedure to show how such a structure may be devised starting from a conventional waveguide Tjunction.

\section{A. Conventional Waveguide T-Junctions}

The microwave and mm-wave T-junction is an important and commonly used component in microstrip circuits such as filters, amplifiers, ring hybrids, and power dividers [26]. Depending on the symmetry of the junction, power can be split in various ways. The $\mathrm{S}$ matrix can be written as

$$
[S]=\left[\begin{array}{lll}
S_{11} & S_{12} & S_{13} \\
S_{21} & S_{22} & S_{23} \\
S_{31} & S_{32} & S_{33}
\end{array}\right]
$$

A conventional matched power splitter has $\left|S_{11}\right|=\left|S_{22}\right|=$ $\left|S_{33}\right|=0$, and $\left|S_{12}\right|=\left|S_{13}\right|=\left|S_{23}\right|=1 / 2$, with reciprocity [26]. However, this assumes frequency invariance, which is untrue in practice. Impedance mismatch further degrades performance. A simple waveguide T-junction operating in its fundamental $\mathrm{TE}_{10}$ mode with equal power split is shown in Fig. 1.a), with its typical response shown in Fig. 1.b) exhibiting strong reflection characteristics. This impedance mismatch can be improved through the introduction of a notch located in an optimized region of the junction, acting as a matching element [27], [28]. Fig. 11b) along with its typical response is shown in Fig. 1.c) with a significantly improved broadband matching. In actual fabrication, this notch is manifested as an inductive post.

Let us switch the input and output ports of a conventional T-junction, so that the input port now becomes an output port, and one of the output ports becomes the new input, as shown in Fig. 1. (a), indicated with Port-Switched Simple T-junction, with its corresponding response shown in Fig. 11.d). Naturally, the power balance is disturbed between the two output ports and poor matching is still present. Next, let us introduce the notch to match this section, where by it is seen that by an optimum design and placement of the notch, an optimal matching is achieved as shown in Fig. 1.e), i.e. Port-Switched Matched Tjunction. This configuration must be compared with a matched T-junction, where the notch was placed symmetrically with respect to the two output ports. For a Port-Switched Matched T-junction, a longitudinal offset $s$ is needed which introduces a transverse asymmetry in the structure, where the transverse plane is $y=0$. Now by controlling the width of Port 3 , power flow in this section can be controlled.

\section{B. Side-Fire LWA}

The port-switched matched T-junction now acts as a power splitter by splitting the input from port 1 to output ports 2 and 3 , respectively, with identical port impedances at port 1 and 2. This represents a fundamental building unit which is self-matched due to optimal design of the notch element. This allows a series cascade of this unit to form an $N$ element periodic structure with $N+2$ ports and a spatial period $p$, which can now be seen simply as a $1: N$ power divider, as illustrated in Fig. 1(a). Since each unit cell element is self-matched using a transversally asymmetric placed notch, the resulting periodic structure also exhibits a broadband matching, whereby its stopband characteristics can be easily controlled. The typical reflection and transmission characteristics for $1: 5$ power divider are shown in Fig. 11(f), where a broadband matching is clearly evident. Due to the periodicity and the travelingwave nature of this structure, the phase of the side ports (2-7) are frequency dependent. For instance, when $p \approx \lambda_{g}$, all the wavefronts that exit these ports are in phase, as shown in the Fig. 1.f). We will refer to this frequency $f_{0}$ as the broadside frequency of the structure. For frequencies, where $p>\lambda_{g}$ $\left(f>f_{0}\right)$, there is a progressive phase delay leading to a phase tilt, which will later will shown to correspond to forward radiation. Finally, for $p<\lambda_{g}\left(f<f_{0}\right)$, there is a progressive phase advance corresponding to backward radiation. These wavefront advances and delays, leading to a phase tilts are further shown in Fig. 1(f) for two specific frequencies.

The cascaded 1: $N$ power divider can now be allowed to have its outputs open to free space readily forming a periodic LWA with beam-scanning capability. Thanks to the selfmatched transversally asymmetric unit cell based on a notch, a seamless transition from the backward to the forward region is possible including broadside radiation. This resulting structure represents a periodic LWA consisting of $N$ unit cells with radiating apertures, allowing free-space radiation from the side walls of a rectangular waveguide operating in its fundamental $\mathrm{TE}_{10}$ mode. Since this structure is compatible with substrate integrated waveguide (SIW) technology, it can now be readily integrated inside a dielectric slab, where the radiation from the aperture array can either be coupled into free-space or confined between top and bottom conductors of the dielectric forming a PPW, as shown in Fig.2 a). If the leaky-wave region outside the main waveguide is confined inside a PPW, the entire structure remains shielded and completely enclosed.

A cell for such a periodic antenna has a stop band at broadside that requires suppression [29]. This behavior can be examined through considering the eigenmodes of the unit cell [30], [31]. The cell schematic is shown in Fig. 2[a). Depending on the dimensions and location of the notch, various dispersion relations of the unit cell may be computed, depending on the symmetries of the unit cell [31], [32]. The eigenmode analysis assumes Floquet (periodic) boundaries on the two faces of the unit cell along the direction of wave propagation. Let us consider the case, when the leaky-wave region is confined inside a PPW, which is terminated with the medium impedance at the far end of the waveguide. While the width of the main waveguide, $a$, the period $p$, the aperture size 

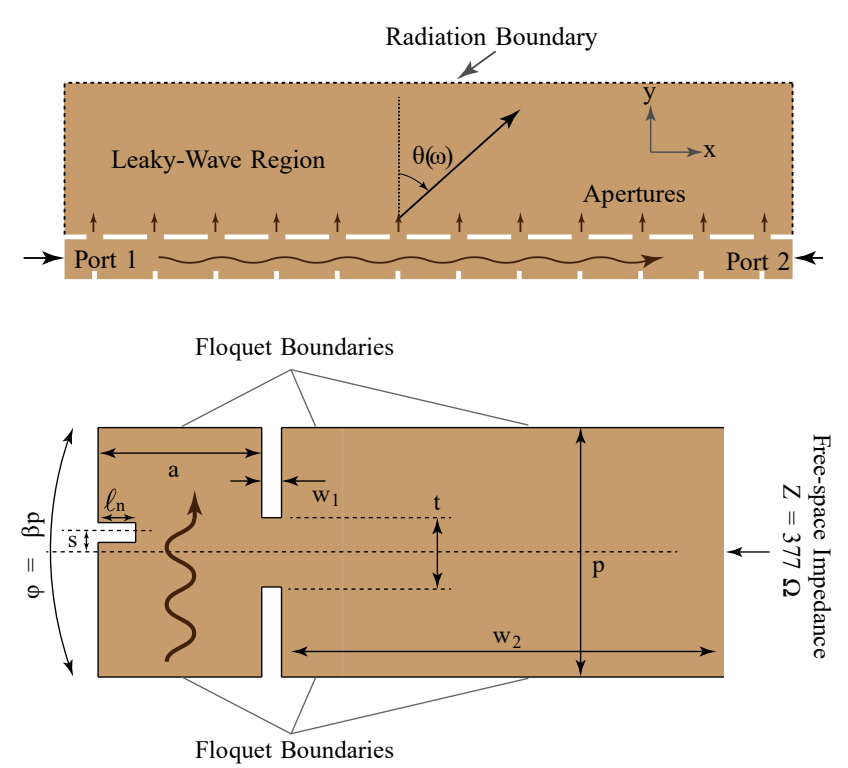

(a)

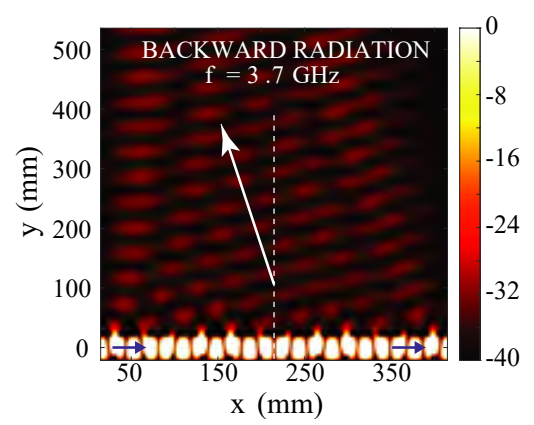

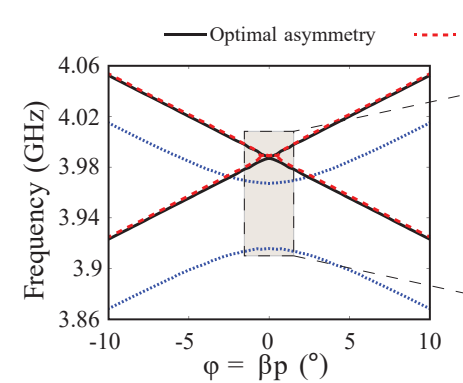

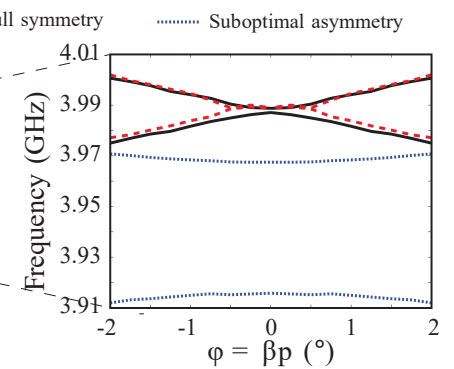

(b)
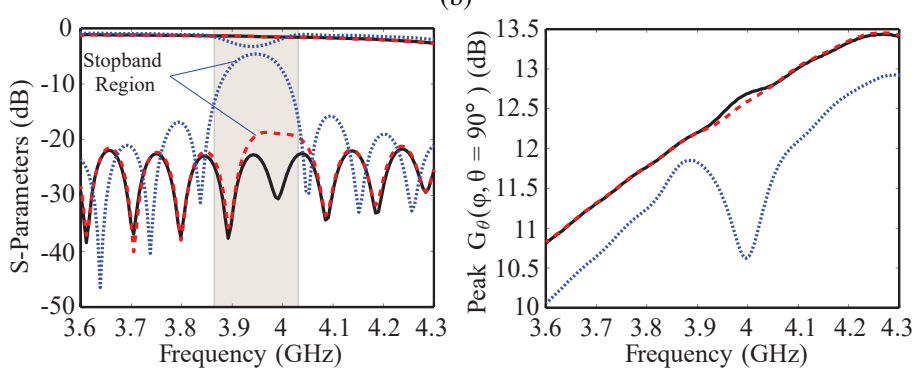

(c)
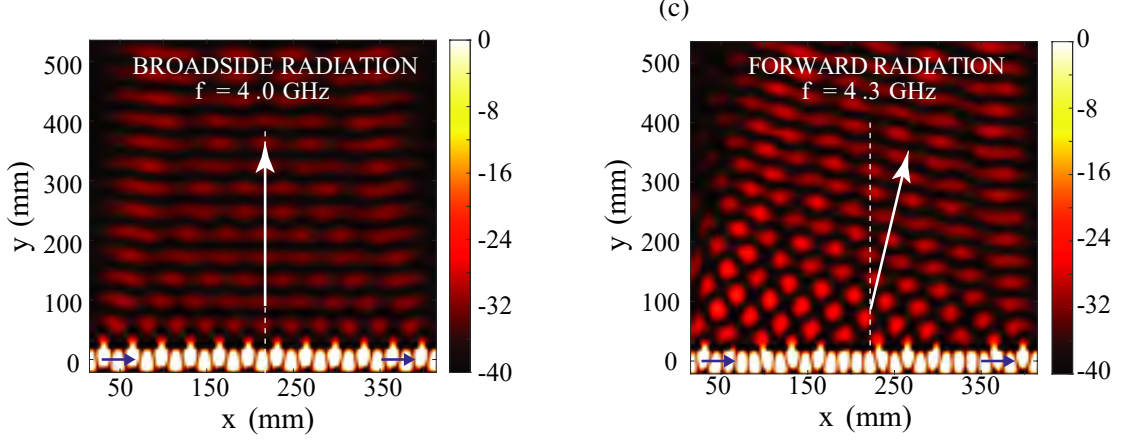

(d)

Fig. 2. The proposed side-fire Leaky-Wave Antenna (LWA) constructed using the $N$-port power divider of Fig. 1 a) A driven-mode LWA structure consisting of 12 cells and the unit cell used for eigenmode analysis in FEM-HFSS, b) the corresponding eigenfrequencies showing three unit cell configurations. c) The driven mode S-parameters and the peak Gain vs frequency. d) Near-fields $E_{z}$ showing the radiated wavefronts in the backward and forward regions, along with that at broadside. Here $p=64 \mathrm{~mm}, t=17.78 \mathrm{~mm}, a=42 \mathrm{~mm}, w_{1}=5.08 \mathrm{~mm}$, and $w_{2}=152.4 \mathrm{~mm}$. For optimal asymmetry: $\ell_{n}=8.64 \mathrm{~mm}$, and $s=0.32 \mathrm{~mm}$. For full symmetry: $\ell_{n}=8.76 \mathrm{~mm}$, and $s=0 \mathrm{~mm}$. For suboptimal asymmetry: $\ell_{n}=2.54 \mathrm{~mm}$, and $s=0.34 \mathrm{~mm}$.

$t$ and width $w_{1}$ are kept constant, the length $\ell_{n}$ and offset $s$ of the notch is varied to study its traveling wave characteristics. The FEM-HFSS computed eigenmodes for various cases of notch configurations is shown in Fig. 2(b), which shows regions of $\beta<0$ corresponding to backward radiation, and $\beta>0$ for foward radiation (for positively sloped branches of the dispersion curves). As expected from the works of Otto et. al. [31], [32], a perfectly closed stopband requires an optimal transversal asymmetry in the unit cell. For sub-optimal asymmetries a large stop-band is present, which prohibits any broadside radiation along $\theta=0^{\circ}$. A fully symmetric unit cell on the other exhibits a frequency balanced condition, but is still a non-optimal condition which is manifested in the flat phase region around the broadside frequency. Ultimately, at the optimal set of notch parameters, the eigenmodes crossover at a single point as shown in shown in Fig. 2(b), and the reflection at this frequency corresponding to broadside is expected to be optimal [33].

To further confirm these eigenmode results, a driven model is analyzed in FEM-HFSS consisting of $N=12$ cells, whose computed frequency dependent transmission/reflection response and far-field peak gain is shown in Fig. 22 (c). As expected, the cell with the optimal asymmetry provides an excellent match throughout the frequency band compared to a suboptimally asymmetric case, while the symmetric unit cell still exhibits a noticeable increase in reflection around broadside in spite of an overall good matching response [32]. Fig. 2(d) finally shows the radiating wavefronts at three different frequencies, clearly showing the wave propagation along backward, broadside and forward directions. Therefore, the proposed side-fire structure represents a fully integrated periodic LWA with complete control over its dispersion relation and the subsequent closure of the stop-band to enable broadside radiation. Exploiting its integrated nature and fully shielded configuration, we will next describe an integrated analog spectrum analyzer for a $60 \mathrm{GHz} \mathrm{mm}$-Wave frequency band.

\section{Proposed Mm-WAVE Spectrum AnAlyzer}

\section{A. Principle}

Leaky-wave antennas have the property of frequency scanning. For a periodic leaky-wave antenna, the aperture spacing 
provides a phase shift between locations of radiation. This spacing is electrically frequency dependent, and therefore provides a phase gradient which enables beam steering. The beam scanning law is described by [20]

$$
\theta(\omega)=\sin ^{-1}\left(\frac{\beta(\omega)}{k_{0}}\right)
$$

where $k_{0}$ is the free space wavenumber, $\beta(\omega)$ is the guided propagation constant, and $\theta(\omega)$ is the beam angle measured from broadside. A real-time spectrum analyzer can be formed using the process of spatial-spectral frequency decomposition, similar to that used in optical prisms [14], [15]. Accordingly, a broadband time domain test signal sent at the input port of a LWA would have its frequency components radiated at particular angles described by (2) with appropriate magnitudes. This enables one to deduce the presence of the frequency components of the signal and their respective magnitudes by inspecting the corresponding far-field radiation patterns. Such a determination of the spectral components of an unknown test signal based on spatial-spectral frequency decomposition principle, is purely analog and thus real-time in nature.

The radiated signals from the LWA are typically measured in the far-field regime of the radiation, which is approximately located at distances greater than $2 d^{2} / \lambda_{0}$, where $d$ is the largest dimension of the antenna aperture, and $\lambda_{0}$ is the free space wavelength. For typical mm-wave leaky-wave antennas which are several wavelengths long, this distance readily exceeds $5 \mathrm{~m}$ when operated around $60 \mathrm{GHz}$. Therefore, to perform spectral analysis using a typical mm-wave LWA, one would need large space to make the measurement. However, this measurement would not be protected from the environment, and thus undesired signal interference would occur between neighbouring devices. Thus shielding is necessary, which makes the proposed side-fire LWA configuration of Fig. 2 particularly attractive. However, the far-field requirement of large system size is still a challenge, since for far-field distances of several meters, the PCB would be impractically large. A potential solution is then to add a physical curvature to the LWA aperture to focus the far-field radiation in the near-field region of the antenna, which is proposed next.

\section{B. Curved Side-Fire LWA}

A solution to the issue of large far-field distances where the beam is found is by introducing a curvature to the antenna [34]. In the case of parabolic reflectors, a parabolic surface allows for a beam focal point where the radiation is concentrated. Depending on the exact surface curvature, the focal location can change, subject to sufficiently small aberrations. [35], [36]. In this sense a convex lens with a close focal point can solve the far distance problem. This principle can be easily applied to the side-fire LWA configuration of Fig. 2, where instead of a linear array, we devise a curved array with desired frequency dependent beam focussing characteristics in its nearfields. Such a structure may behave like a lens, allowing the beam location to be within the range of a typical PCB lengths, while preserving the spatial-spectral decomposition information needed for spectral analysis.

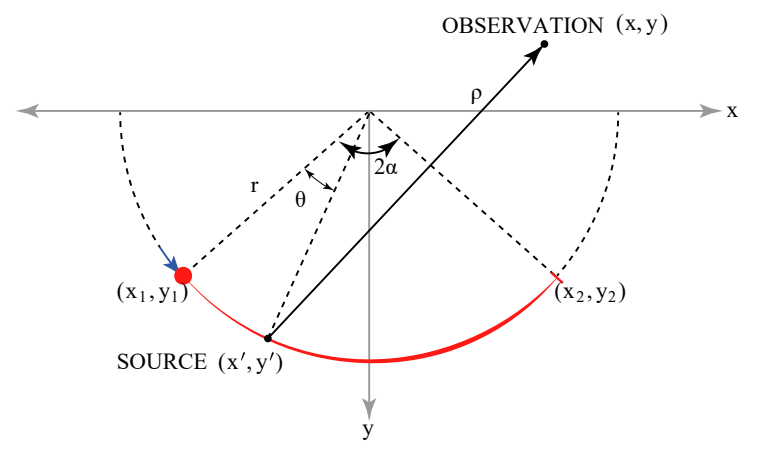

(a)

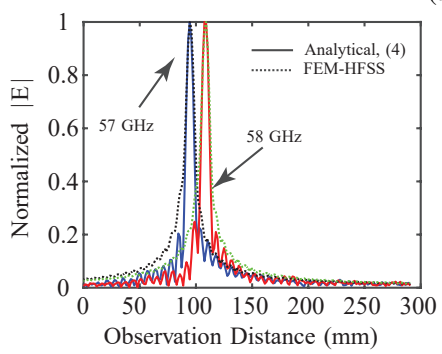

(b)

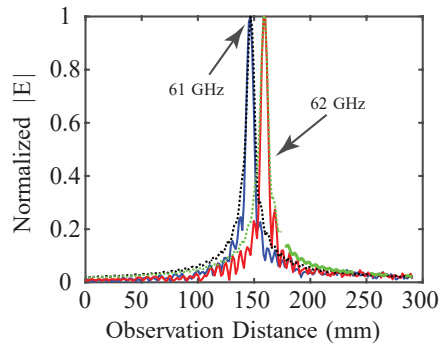

(d)

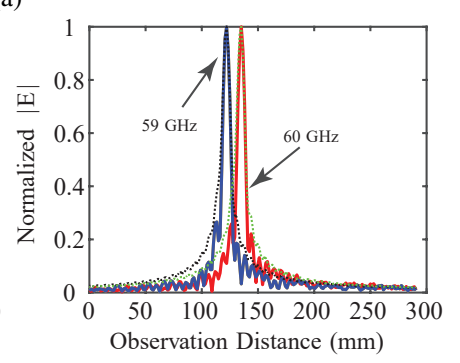

(c)

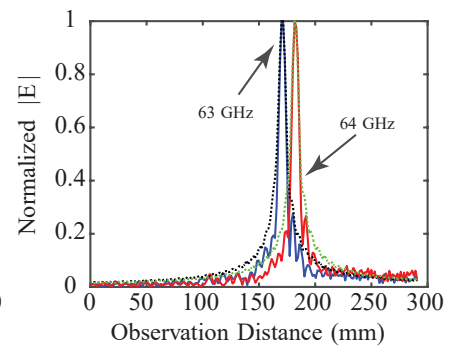

(e)
Fig. 3. Analytical method to compute the near-fields of a side-fire antenna. a) Illustration of a curved side-fire antenna showing the source and observation points. (b)-(e) Comparison of electric fields calculated along a line at the focal point using the wavenumber computed from FEM-HFSS eigenmode analysis with full-wave electric fields along a line at the focal distance $(r=56 \mathrm{~cm})$.

With a determined antenna structure and periodic aperture producing a specified per unit length propagation constant $\beta(\omega)$, a numerical model to calculate the radiation and focussing properties is needed to understand and model this structure. Consider a uniform circular LWA as shown in Fig. 3(a), of an angular span $2 \alpha$ and a radius $r$, excited from the left. The field variation along the $z$-axis is assumed to be zero, i.e. a $2 \mathrm{D}$ problem is assumed. Each point on this LWA can be assumed to be a line source $J_{z}\left(x^{\prime}, y^{\prime} ; \omega\right)$ which is radiating with a certain phase accumulated along the LWA. Each source coordinate follows the following parametric curve

$$
x^{\prime 2}+y^{\prime 2}=r^{2} .
$$

The total radiated electric field in the $x-y$ plane can be analytically expressed as [16]

$$
\mathbf{E}(x, y)=\int_{-r \sin \alpha}^{r \sin \alpha} J_{z}\left(x^{\prime}, y^{\prime}\right) G\left(x^{\prime}, y^{\prime} ; x, y\right) d x^{\prime} \hat{z}
$$

where $G\left(x^{\prime}, y^{\prime} ; x, y\right)$ is the Green's function. This integral represents the contribution of each source at the observation 


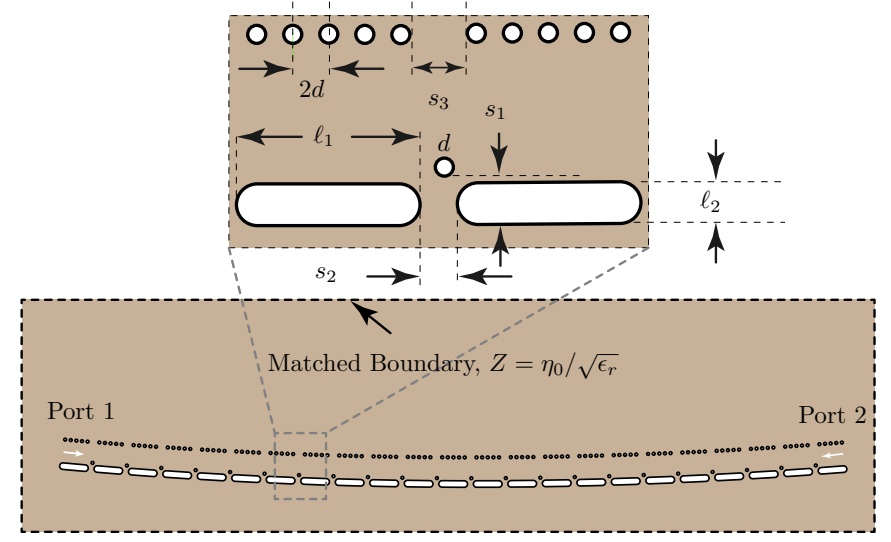

(a)

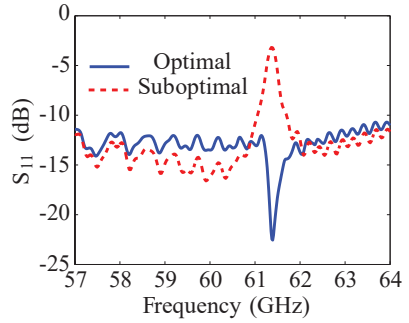

(b)

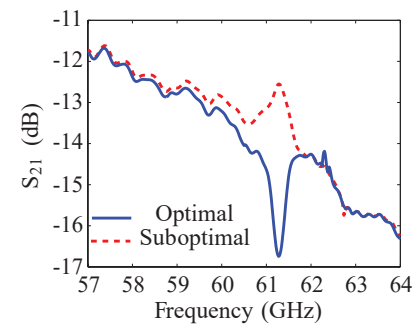

(c)
Fig. 4. Integrated Side-Fire LWA design. (a) FEM-HFSS cell model of the curved LWA based on substrate integration, showing the unit cell design parameters. Here $d=0.34 \mathrm{~mm}, s_{3}=1.08 \mathrm{~mm}, \ell_{1}=3.46 \mathrm{~mm}, \ell_{2}=$ $0.8 \mathrm{~mm}, \epsilon_{r}=2.2 \mathrm{~mm}$, and $s_{1}=1.1 \mathrm{~mm}$. (b) $S_{11}$ and (c) $S_{21}$ of the curved antenna with optimal via $s_{2}=0.1 \mathrm{~mm}$ compared to suboptimal fully symmetric via $s_{2}=0 \mathrm{~mm}$.

point $(x, y)$ along the entire extent of the antenna. For a 2D problem we have a Hankel's function of the second kind, given by

$$
G\left(x^{\prime}, y^{\prime} ; x, y\right)=-\frac{\omega \mu_{0}}{4} H_{0}^{(2)}(k \rho),
$$

with

$$
\rho\left(x^{\prime}, y^{\prime} ; x, y\right)=\sqrt{\left(x-x^{\prime}\right)^{2}+\left(y-y^{\prime}\right)^{2}} \hat{\rho},
$$

where $\rho$ represents the vector from the source to the observation point, as illustrated in Fig. 3. a). Finally, the position dependent current source is assumed to take the following form:

$$
J_{z}\left(x^{\prime}, y^{\prime}\right) \approx e^{-j \beta s}
$$

where the length $s=r \theta$ represents the arc length from $\left\{\left(x_{1}, y_{1}\right)-\left(x^{\prime}, y^{\prime}\right)\right\}$ and can be easily shown to be

$$
s=r \cos ^{-1}\left\{1-\frac{\left(x^{\prime}-x_{1}\right)^{2}+\left(y^{\prime}-y_{1}\right)^{2}}{2 r^{2}}\right\}
$$

Therefore, substituting (5), (7) inside (4) leads to the analytical form of the field anywhere in the observation area.

Next, an integrated spectrum analyzer based on a side-fire LWA configuration of Fig. 2 with a $1 \mathrm{GHz}$ spatial resolution operating from 57-64 GHz was designed and simulated in FEM-HFSS, as shown in Fig. 4(a). To be compatible with standard PCB fabrication, the vertical solid walls of Fig. 2 are realized using a metallized via array as typically done in SIW implementations, and a curvature is introduced. An isolated via is used to emulate a notch and its precise placement is obtained to close the stop-band following the analysis of Fig. 2 to obtain the required asymmetry. Fig. 4(b,c), further shows the simulated S-parameters for the case of an optimal and suboptimal asymmetry, clearly showing broadband matching within the bandwidth of interest, i.e. 57-64 GHz.

Next, an analytical model is built for this side-fire configuration, where its propagation constant $\beta(\omega)$ corresponding to the $n=-1$ space harmonic is approximated as

$$
\beta(\omega) \approx \sqrt{\left(\frac{\omega}{c}\right)^{2} \epsilon_{r}-\left(\frac{\pi}{a}\right)^{2}}-\frac{2 \pi}{p} .
$$

This analytical result closely approximates the full-wave computed dispersion relation extracted from an eigenmode simulation of a single cell, and is thus used due to its simplicity to estimate beam-scanning of the structure in the near-field region of the antenna. Using (5), (7) and (9) in (4), the radiated near-fields were calculated along a line at the focal distance $r$, and compared with those obtained using the full-wave HFSS model, as shown in Fig. 3 (b-d) for several frequencies. An excellent match is seen for all frequencies which successfully validates the model. While the HFSS model is rigorously correct, it is electrically large and computationally expensive. The analytical model on the other hand, captures the fundamental behavior of the structure using a much simpler model based on array of line sources, while providing a reasonably good accuracy. It thus represents an ideal method for an efficient design and fast analysis of a side-fire LWA based spectrum analyzer.

\section{EXPERIMENTAL DEMONSTRATION}

In this section, two experimental demonstrations will be presented: 1) A straight integrated side-fire LWA radiating into free space and, b) a curved side-fire antenna radiating inside an integrated PPW acting as a mm-wave spectrum analyzer.

\section{A. Straight Side-Fire LWA}

Fig. 5(a) shows a photograph of a 15 cell straight sidefire LWA operating around the $60 \mathrm{GHz}$ band, following the configuration of Fig. 4(a), except with a straight cell. The matching via is optimized in simulations to close the stopband using the nominal permittivity of the dielectric specified by the manufacturer. A transition is further designed to excite the LWA antenna using a standard WR-15 waveguide, as shown in the input and output ends of the LWA structure. One side of the antenna is left open to free-space with a width of the PPW controlling the amount of radiation along the $-y$ direction. Fig. 5(b-c) shows the measured S-parameters compared with FEM-HFSS. While good matching is observed in measurements as a result of optimal via placement, a slight increase in the reflection is also observed along with appreciable mismatch with the simulated response. This mismatch between simulation and measurement can be attributed to the the unknown material properties at $60 \mathrm{GHz}$, and the tolerances in fabrication, especially considering that the response is particularly sensitive to the matching via location. 


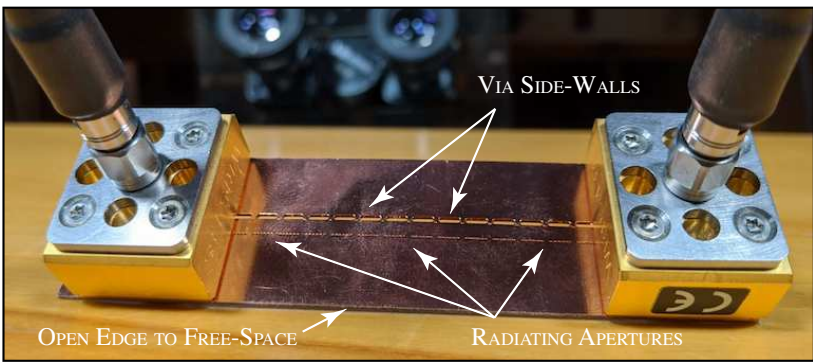

(a)

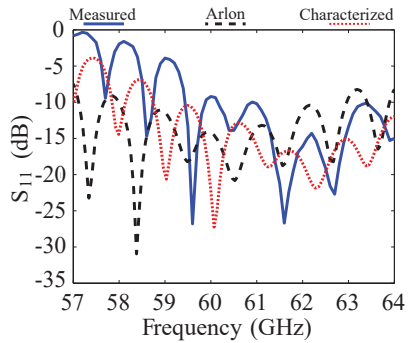

(b)

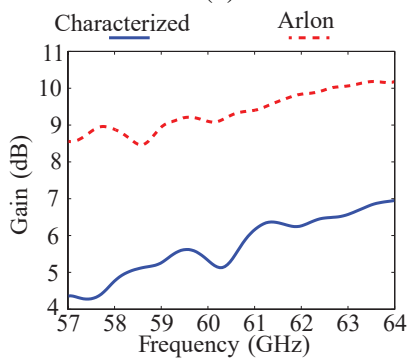

(d)

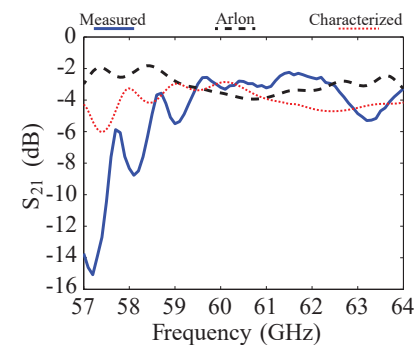

(c)

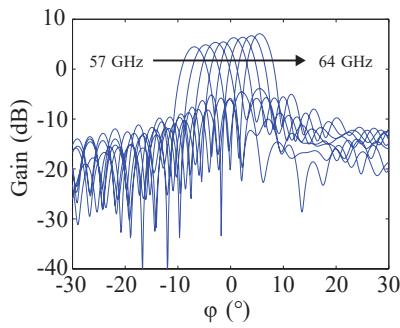

(e)
Fig. 5. Experimental demonstration of a straight side-fire LWA radiating into free-space. (a) Fabricated antenna prototype ( $N=15$ cells). (b) $S_{11}$ of the antenna in FEM-HFSS with Arlon and the resonator characterized material compared to the VNA measurement. Here the stopband is closed at $61 \mathrm{GHz}$ (broadside). (c) $S_{21}$ of the antenna in FEM-HFSS with Arlon and the resonator characterized material compared to the VNA measurement. (d) Simulated gain as a function of frequency for the antenna with the characterized material and Arlon DiClad 880. (e) Radiation patterns of the simulated antenna with characterized material in $1 \mathrm{GHz}$ steps over the band of interest.

To estimate the material properties, a straight waveguide resonator was also fabricated, which was then used to estimate the effective dielectric constants of the Arlon DiClad 880 material used in fabrication. The resonator results are presented in the Appendix, and the extracted dielectric permittivity and loss tangent are found to be $\approx 2.08$ and 0.003 , respectively, compared to the design values of $\epsilon_{r}=2.2$ and $\tan \delta=0.0009$. The fabricated straight side-fire LWA was then re-simulated with the new material parameters, and the new S-parameters are shown in Fig. 5(b-c), improving the agreement with the measurements. Importantly, a frequency shift of almost $1.6 \mathrm{GHz}$ is observed, with a new broadside frequency of $f_{0}=60.7 \mathrm{GHz}$ (observed in HFSS with peak radiation at $\theta=0^{\circ}$ ). The resulting frequency dependent gain and the simulated radiation patterns are shown in Fig. 5.de) showing the seamless radiation from backward to forward regions including broadside at $60.7 \mathrm{GHz}$, as expected. Due to an actual higher loss tangent of the dielectric, the gain is seen to be significantly dropped with a slight drop at broadside, while maintaining a monotonically increasing trend, as expected.

\section{B. Integrated Spectrum Analyzer}

Next, an integrated spectrum analyzer based on a sidefire LWA will be demonstrated. Fig. 6(a) shows the general schematic of the proposed system. A curved side-fire LWA of Fig. 4 with larger number of cells and input/output transitions to standard WR-15 waveguides is placed on the far right of the dielectric layer, where Port 1 is intended as the input to the system. the leakage from the side apertures is maintained inside the PPW structure, due to the beam-scanning property, the spatial-spectral decomposition is achieved in the $x-y$ plane, with broadside along $-x$ and $x<0$ and $x>0$ as the backward and forward radiation regions, respectively. The system is designed to operate between $57-64 \mathrm{GHz}$, with a frequency resolution of $1 \mathrm{GHz}$.

To capture the power radiated along different angles, an integrated horn array is chosen for the sake of a proof of concept experiment (detailed dimensions are provided in Tab. I). To achieve a $1 \mathrm{GHz}$ resolution over the $57-64 \mathrm{GHz}$ band, 8 horns for each $1 \mathrm{GHz}$ frequency step are needed where the transmission in the form of S-parameters can be measured. The receivers are waveguide fed, and thus must be spaced to allow room for WR-15 flanges, which have a diameter of $19.05 \mathrm{~mm}$. The unknown physical dimensions to achieve this resolution are: the horn spacing, radius of curvature and the angular span of the side-fire antenna. To determine these parameters, a simple parametric analysis of Fig. 3 a) is performed. Fig. 7(a) shows the maximum scan range (using peak amplitudes of the focussed beam) over 57-64 GHz for each $r$ at a fixed frequency $f$, for instance. Knowing the required separation between the horn receivers of $\approx 12 \mathrm{~mm}, r$ is set at $56 \mathrm{~cm}$. Next, Fig. 7(b) shows the proper antenna arc length for a given radius of $56 \mathrm{~cm}$ to produce a sufficiently narrow beam, less than the 3 -dB beamwidth of the horn. It was then set at $2 \alpha=30^{\circ}$. This would then allow 1 $\mathrm{GHz}$ resolution while preventing electromagnetic coupling to neighbouring receivers which could compromise the spectral decomposition.

Fig. 6.b) shows the FEM-HFSS simulated power transmission between the input port 1 and the various horn receiver ports. A linear frequency scanning can be seen across the ports, with clear separation between the power received by adjacent ports. Direct incidence of the beam on a port results in a transmission of more than $-15 \mathrm{~dB}$. Neighbouring ports in such a case would have less than $-30 \mathrm{~dB}$ in transmission. To this end, a nominal frequency resolution of $1 \mathrm{GHz}$ is readily shown. Fig. 6 (c) further shows the near-field distribution inside the PPW for various frequencies, which are being received by different horn receivers demonstrating the frequency scanning behavior of the side-fire antenna. In addition, extra radiation beams are also observed in the forward region, which are analogous to grating lobes in regular antenna arrays.

To understand the nature of these grating lobes, the dispersion diagram of the side-fire LWA must be inspected as 


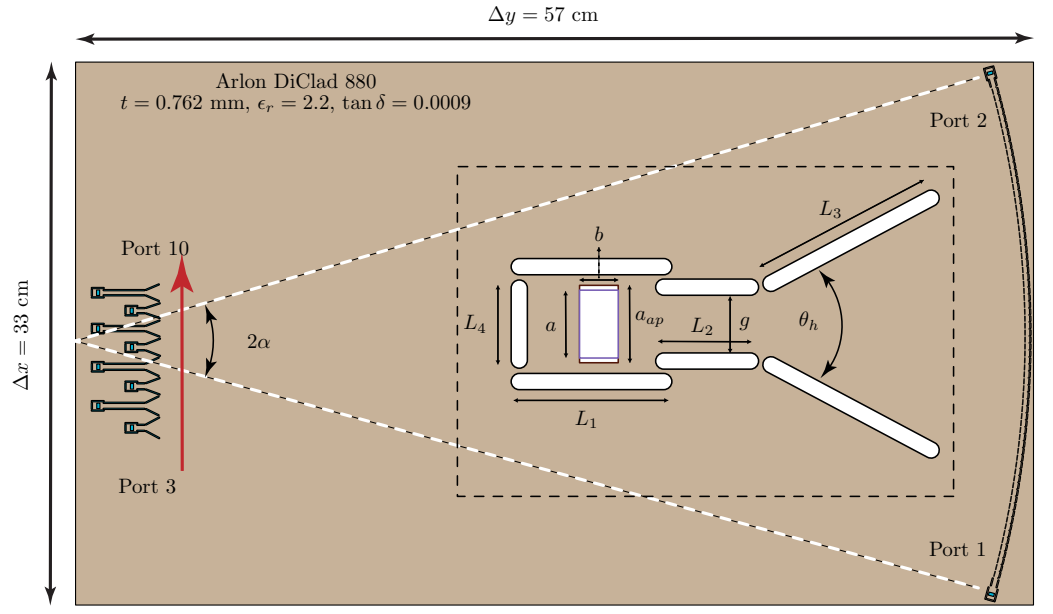

(a)

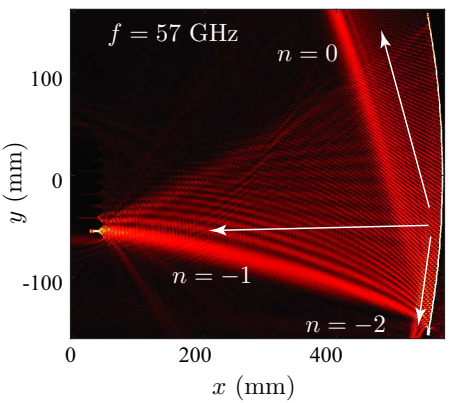

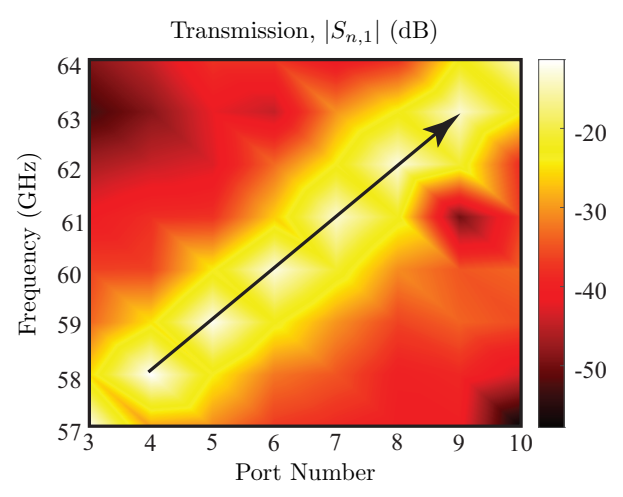

(b)
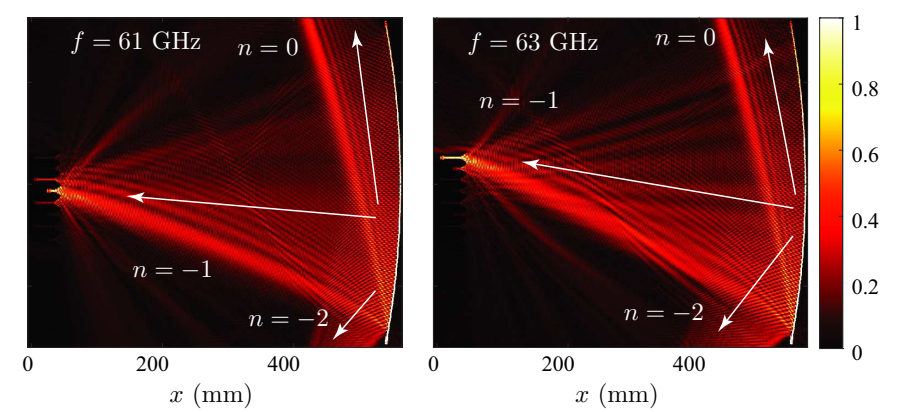

(c)

Fig. 6. FEM-HFSS modeling of the integrated side-fire LWA based spectrum analyzer. (a) Schematic model showing an array of integrated horn receivers with $\Delta x=570 \mathrm{~mm}$ and $\Delta y=323 \mathrm{~mm}$, and the curved side-fire LWA consisting of $N=72$ unit cells. (b) Interpolated transmission into the receiving horn ports. (c) Normalized $\left|E_{z}\right|$ plots from HFSS of the spectrum analyzer showing the beam focusing. All horns and aperture dimensions shown in Tab. 1.

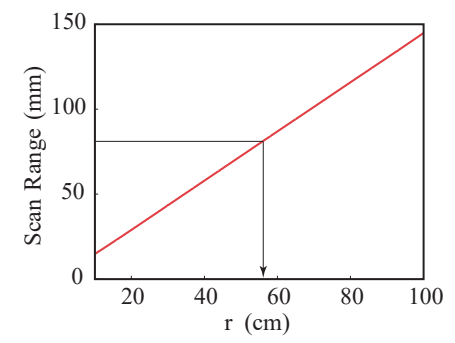

(a)

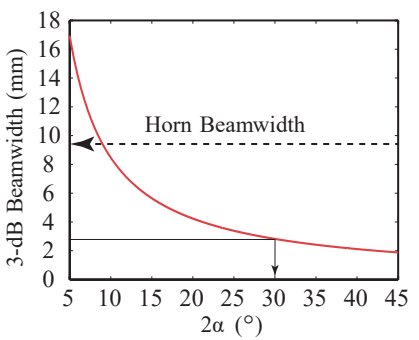

(b)
Fig. 7. Procedure to determine the radius of curvature of the curved side-fire LWA and the arc length $2 \alpha$ to achieve a frequency resolution of $1 \mathrm{GHz}$ within the operating bandwidth of $57 \mathrm{GHz}-64 \mathrm{GHz}$. (a) Beam scan variation with $r$ at $f=60.7 \mathrm{GHz}$. b) Beamwidth as a function of angular span of the LWA for $r=56 \mathrm{~cm}$.

shown in Fig. 8. For the designed substrate permittivity and antenna dimensions (curvature, width, aperture period), more than one fast-wave harmonic appears in the radiative region. Since radiation is in the substrate, the slope of the light line is increased by a factor of $1 / \sqrt{\epsilon_{r}}$ compared to that in freespace. As a result, the fundamental mode does not leave the radiative region since it is parallel to the light line. Therefore, the fundamental mode always remain inside the fast-wave region and radiates in the forward direction. At the same time, other spatial harmonics, particularly $n=\{-1,-2\}$ harmonics, also radiate within the band of interest [24] [21]. They appear

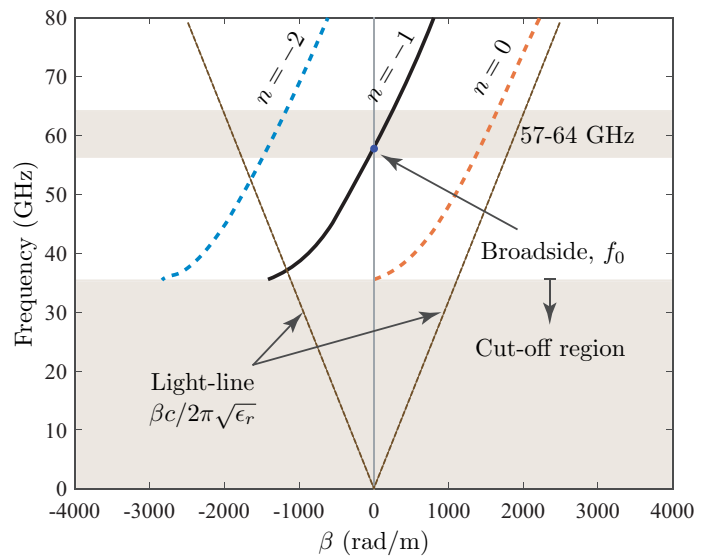

Fig. 8. Dispersion diagram of the conformal leaky-wave antenna illustrating the issue of multi-beam radiation within the fast-wave region.

as extra beams in the full-wave simulated fields of Fig. 6(c), where $n=-1$ are the ones captured by the horn receivers. While these spurious grating lobes represent unwanted power loss through radiation, the dominant power is still found to be contained in the $n=-1$ harmonic sufficient for the current purpose of spectrum measurement.

The side-fire based integrated spectrum analyzer is next fabricated and some pictures of the prototype and the experimental setup is shown in Fig. 9(a-b). Fig. 9(a) shows 


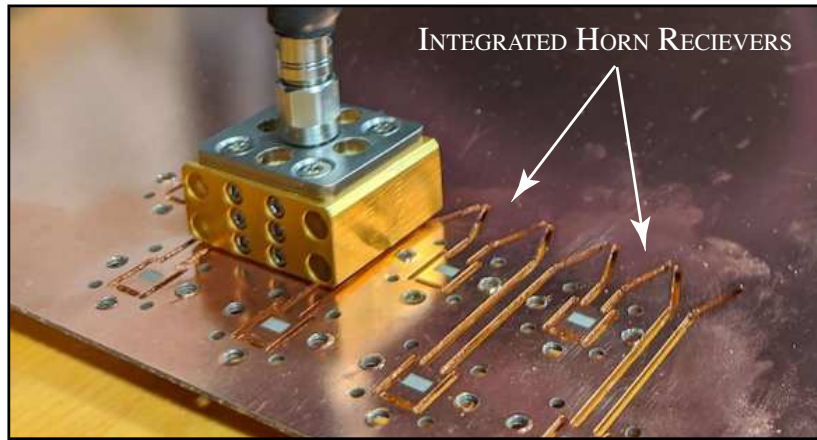

(a)

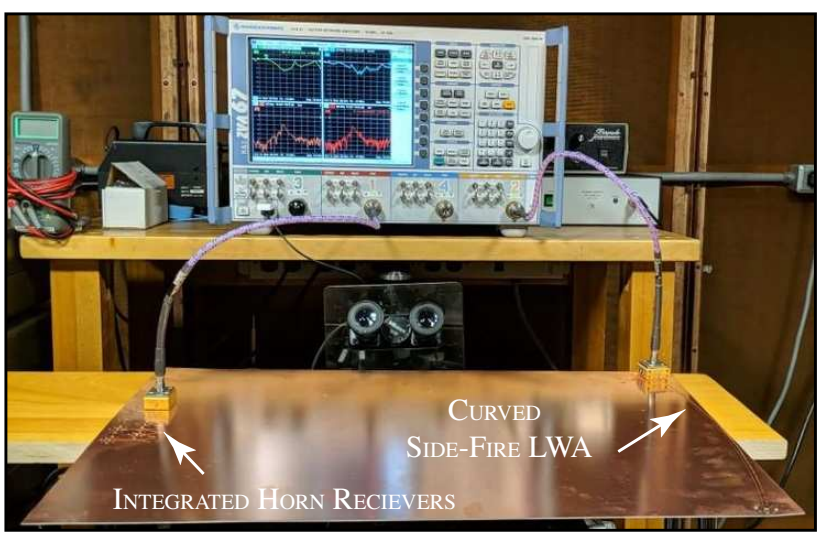

(b)

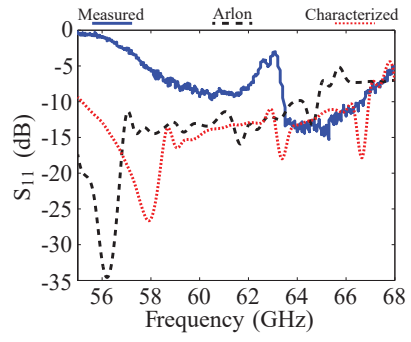

(c)

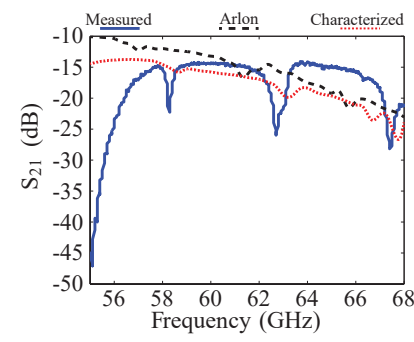

(d)
Fig. 9. Experimental setup and results of the fabricated spectrum analyzer. (a) Zoomed in view of the receiver horn array during S-parameter measurement. b) S-Parameter measurement of the VNA. (c) Measured $S_{11}$ and (d) $S_{21}$ of the spectrum analyzer compared to simulation with Arlon DiClad 880, and in simulation fitted material parameters (Appendix A).

the array of integrated horns on the receiver side connected to a standard WR-15 waveguide to a V-band coax cable. The integrated spectrum analyzer is next measured using a Vector Network Analyzer as shown in Fig. 9(b), where different measurements are taken across various horn ports. The S-parameters of the curved side-fire antenna is shown in Fig. 9(c,d) where in spite of an intended well-matched design in simulations, relatively larger reflections are observed, particularly around $62 \mathrm{GHz}$. This indicates a stop-band region and a high frequency shift in the response, as the broadside frequency was around $60 \mathrm{GHz}$. Transmission on the other hand stays around $-15 \mathrm{~dB}$. The spectrum analyzer was then resimulated in HFSS using the newly characterized parameters of the dielectrics (provided in Appendix), and the resulting Sparameters are shown also in Fig. 9.(c,d). The frequency shift is clearly captured as seen in $S_{11}$ and an increase in reflection is observed in spite of being practically acceptable. However an appreciable difference between measurements and simulations still remains. This suggests that small variations in tolerances and material properties may significantly accumulate to give rise to large errors, since this integrated spectrum analyzer is still electrically large to the order of about $120 \lambda$.

Next, the transmission across various horn ports receivers from the input LWA port is measured and are shown in Fig. 10. A clear shift in the transmission peak is observed across frequency as different horn receivers are measured, confirming the frequency scanning behaviour. This is attributed to the lower permittivity of the actual material. The measurements results are next compared with the characterized material response in FEM-HFSS and a very good agreement is observed where not only the frequency shift but the transmission levels are correctly reproduced. The spectrum analyzer thus operate between $59 \mathrm{GHz}-66 \mathrm{GHz}$ (instead of $57 \mathrm{GHz}-64 \mathrm{GHz}$ ), with a resolution of approximately $1 \mathrm{GHz}$ and an average transmission level of about $-20 \mathrm{~dB}$. The proposed side-fire LWA based integrated spectrum analyzer is thus successfully demonstrated.

\section{CONClusions \& Future WORK}

An analog, low-profile and shielded spectrum analyzer has been proposed for operation at mm-wave frequencies around the $60 \mathrm{GHz}$ band based on a novel side-fire LWA configuration. The proposed side-fire LWA has been systematically developed from a conventional 3-port waveguide T-junction which has been modified to a LWA unit cell with an internal matching mechanism to suppress stop-band to enable broadside radiation. The internal mechanism is achieved by introducing a transversal asymmetry of the unit cell, by placing a notch inside the waveguide, which results in a seamless leaky-wave radiation from backward to forward region including broadside. The proposed antenna is fully compatible with SIW technology resulting in a low-profile and is compatible with standard PCB fabrication. The resulting periodic sidefire antenna radiates in the plane of the antenna, whereby the leakage power be either be allowed to radiate in free-space or kept confined inside a PPW structure. The proposed side-fire structure thus can be completely shielded, which is particularly attractive for devising an analog broadband spectrum analyzer. Such a system has been demonstrated around the $60 \mathrm{GHz}$ band, where a convex side-fire antenna has been used to focus the radiated beams in the near-field of the structure to make the entire system more compact, operating between $59-66 \mathrm{GHz}$ in experiments with a $1 \mathrm{GHz}$ resolution. Furthermore, a simple mathematical model consisting of an array of point sources has also been proposed which is found to faithfully reproduce the beam-scanning characteristics of the curved side-fire LWA in the near-field, providing a fast design tool to engineer such a system.

The proposed side-fire antenna also provides a different interpretation of a LWA, where a periodic leaky-wave structure may be seen as a broadband multi-port power divider, whose outputs are connected to radiating apertures. The proposed spectrum analyzer furthermore offers several system features 


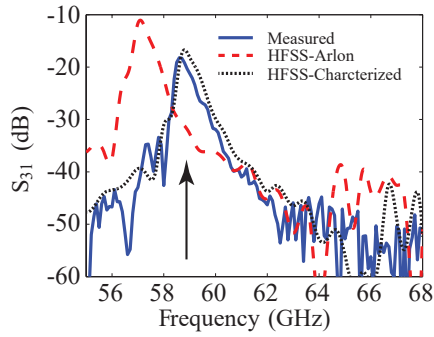

(a)

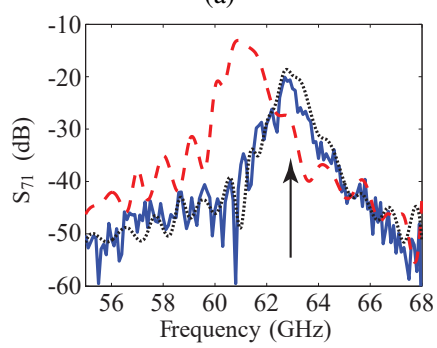

(e)

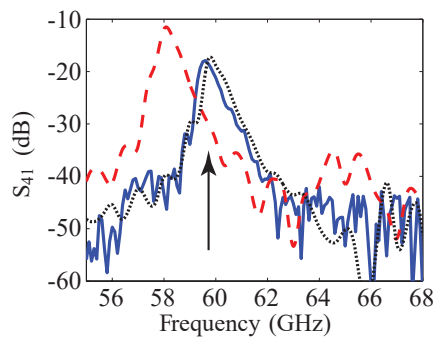

(b)

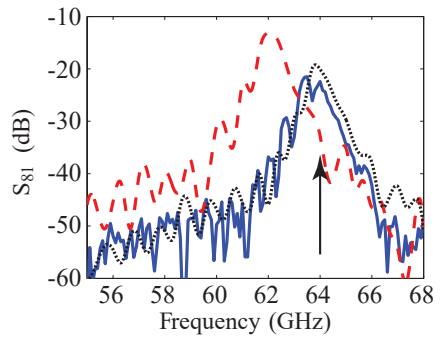

(f)

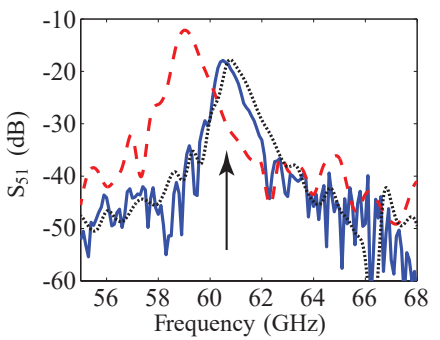

(c)

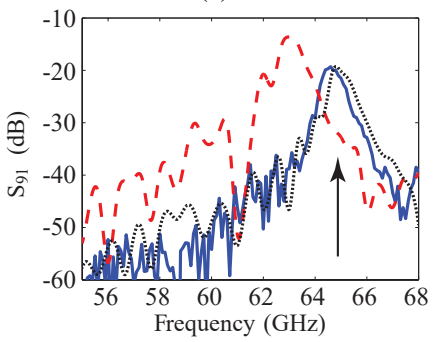

(g)

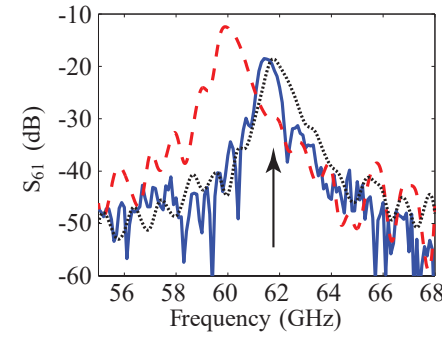

(d)

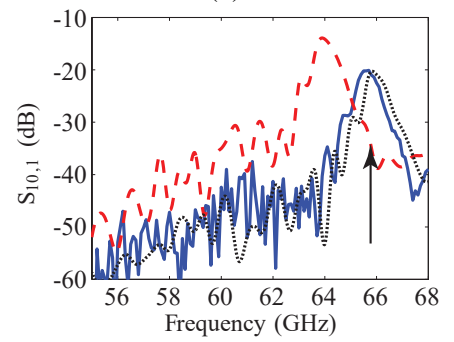

(h)

Fig. 10. Measured S-parameters of the integrated spectrum analyzer of Fig. 9 demonstrating the spatial-spectral decomposition. Adjusted $\epsilon_{r}=2.08$ from nominal $\epsilon_{r}=2.2$ for Arlon DiClad 880. Adjusted $\tan \delta=0.003$ from nominal $\tan \delta=0.0009$ for Arlon DiClad 880 following the material characterization given in the Appendix. (a)-(f) Correspond to ports 3-10, respectively.

TABLE I

PORT AND HORN DIMENSIONS.

\begin{tabular}{|c|c|c|c|c|c|c|c|c|c|c|}
\hline Dimension & Port 1 & Port 2 & Port 3 & Port 4 & Port 5 & Port 6 & Port 7 & Port 8 & Port 9 & Port 10 \\
\hline$a(\mathrm{~mm})$ & 3.7592 & 3.7592 & 3.7592 & 3.7592 & 3.7592 & 3.7592 & 3.7592 & 3.7592 & 3.7592 & 3.7592 \\
\hline$b(\mathrm{~mm})$ & 1.8796 & 1.8796 & 1.8796 & 1.8796 & 1.8796 & 1.8796 & 1.8796 & 1.8796 & 1.8796 & 1.8796 \\
\hline$a_{a p}(\mathrm{~mm})$ & 3.3019 & 3.3019 & 3.2965 & 3.2965 & 3.2965 & 3.2965 & 3.2965 & 3.2700 & 3.2700 & 3.2700 \\
\hline$L_{1}(\mathrm{~mm})$ & 7.807 & 7.807 & 7.807 & 7.807 & 7.807 & 7.807 & 7.807 & 7.807 & 7.807 & 7.807 \\
\hline$L_{2}(\mathrm{~mm})$ & 5 & 5 & 5 & 25 & 5 & 25 & 5 & 25 & 5 & 25 \\
\hline$L_{3}(\mathrm{~mm})$ & 0 & 0 & 9.6 & 9 & 9.3 & 9 & 9.6 & 9.3 & 9.3 & 9 \\
\hline$L_{4}(\mathrm{~mm})$ & 4.2843 & 4.2843 & 4.2843 & 4.2843 & 4.2843 & 4.2843 & 4.2843 & 4.2843 & 4.2843 & 4.2843 \\
\hline$g(\mathrm{~mm})$ & 2.81 & 2.81 & 2.81 & 2.81 & 2.81 & 2.81 & 2.81 & 2.81 & 2.81 & 2.81 \\
\hline$\theta_{h}\left(^{\circ}\right)$ & 0 & 0 & 56 & 52 & 40 & 50 & 42 & 42 & 37 & 46 \\
\hline
\end{tabular}

and benefits. The system is broadband in nature due to its wide-band radiating property of the stop-band suppressed sidefire LWA, directly operating at mm-wave frequencies based on SIW technology. It is a fast analog system minimizing digital computations compared to a fully digital system, except at the data acquisition stage. The frequency resolution of the system depends on the length of the side-fire antenna. This resolution can further be improved by optimizing the length of the antenna and controlling the per-unit-length leakage from the unit cell, while keeping the overall size of the analyzer the same. The low-profile and compact size of the spectrum analyzer represents a bench-top type system that can be placed on standard equipments with no electromagnetic interference due to its fully-shielded configuration. Thus the proposed integrated side-fire antenna and the associated spectrum analyzer system represent an attractive solution for next generation $5 \mathrm{G}$ $\mathrm{mm}$-wave systems in the area of wireless communication and instrumentation, for instance.

\section{APPENDIX: MATERIAL ChaRACTERIZATION}

Given the sensitivity of the devices to electrical material parameters such as $\epsilon_{r}$ and $\tan \delta$ (particularly the beam location of the spectrum analyzer considering the large focal length) a material characterization of the panel used to fabricate the devices was performed. This was done by fabricating a resonator and measuring its frequency dependent transmission $S_{21}$, as shown in Fig. 11. The location and magnitudes of the peaks can then be fitted by running additional simulations of the resonator with new values of $\epsilon_{r}$ and $\tan \delta$. These values which match measurement most closely provide a more accurate characterization of the panel material beyond the standard values of Arlon DiClad 880 provided in HFSS. However, given that the frequency dependence of the material parameters varies from what is predicted in software, over a large bandwidth the curves cannot be perfectly fit over the band, hence some mismatch will occur. The curves were fit best to match over the band of interest $(57-64 \mathrm{GHz})$ and the extracted effective material parameters were found to be $\epsilon_{r}=2.08$ and $\tan \delta=0.003$.

\section{REFERENCES}

[1] S. Farzaneh, A. K. Ozturk, A. R. Sebak, and R. Paknys, "Antenna-pattern measurement using spectrum analyzer for systems with frequency translation [measurements corner]," IEEE Antennas Propag. Mag., vol. 51 no. 3, pp. 126-131, 2009.

[2] M. G. Sánchez, M. P. Táboas, and E. L. Cid, "Millimeter wave radio channel characterization for 5G vehicle-to-vehicle communications," Measurement, vol. 95, pp. 223-229, 2017. 


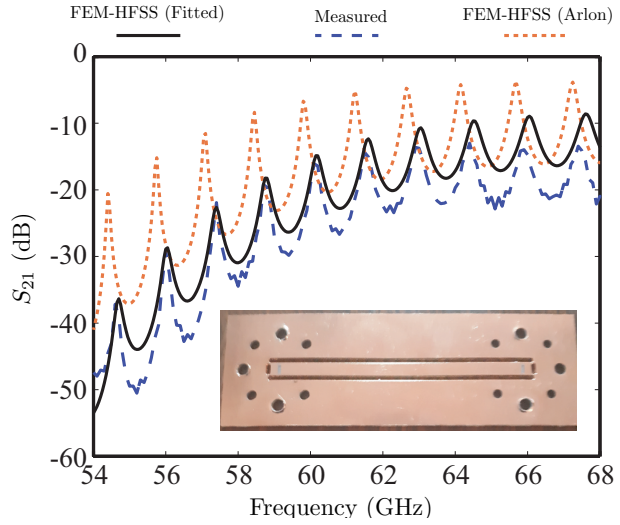

Fig. 11. Fabricated waveguide resonator for effective material characterization showing $S_{21}$. Arlon DiClad $880\left(\epsilon_{r}=2.2\right.$ and $\left.\tan \delta=0.0009\right)$, and FEM-HFSS with fitted material $\left(\epsilon_{r}=2.08\right.$ and $\left.\tan \delta=0.003\right)$. Resonator length and width are $50 \mathrm{~mm}$ and $2.5 \mathrm{~mm}$, respectively.

[3] T. Kawamura, H. Shimotahira, and A. Otani, "Novel tunable filter for millimeter-wave spectrum analyzer over $100 \mathrm{GHz}$," IEEE Trans. Instrum. Meas., vol. 63, no. 5, pp. 1320-1327, 2014.

[4] G. Bergland, "Fast Fourier transform hardware implementations-an overview," IEEE Trans. Audio Electroacoust., vol. 17, no. 2, pp. 104$108,1969$.

[5] T. S. Rappaport, Y. Xing, G. R. MacCartney, A. F. Molisch, E. Mellios, and J. Zhang, "Overview of millimeter wave communications for fifthgeneration $(5 \mathrm{G})$ wireless networkswith a focus on propagation models," IEEE Trans. Antennas Propagat., vol. 65, no. 12, pp. 6213-6230, 2017.

[6] A. Ghosh, "The 5G mmwave radio revolution," Microw. J., vol. 59, no. 9, pp. 22-36, 2016.

[7] J. Karjalainen, M. Nekovee, H. Benn, W. Kim, J. Park, and H. Sungsoo, "Challenges and opportunities of mm-wave communication in $5 \mathrm{G}$ networks," in 2014 9th international conference on cognitive radio oriented wireless networks and communications (CROWNCOM). IEEE, 2014 pp. 372-376.

[8] H. G. de Chatellus, L. R. Cortés, and J. Azaña, "Optical real-time Fourier transformation with kilohertz resolutions," Optica, vol. 3, no. 1, pp. 1-8, 2016.

[9] B. E. Saleh and M. C. Teich, Fundamentals of photonics. John Wiley \& Sons, 2019

[10] J. W. Goodman, Introduction to Fourier optics. Roberts and Company Publishers, 2005

[11] M. Shirasaki, "Large angular dispersion by a virtually imaged phased array and its application to a wavelength demultiplexer," Opt. Lett. vol. 21, no. 5, pp. 366-368, 1996.

[12] M. A. Laso, T. Lopetegi, M. J. Erro, D. Benito, M. J. Garde, M. A. Muriel, M. Sorolla, and M. Guglielmi, "Real-time spectrum analysis in microstrip technology," IEEE Trans. Microw. Theory Tech., vol. 51, no. 3, pp. 705-717, 2003.

[13] X. Wang, A. Akbarzadeh, L. Zou, and C. Caloz, "Real-time spectrum sniffer for cognitive radio based on Rotman lens spectrum decomposer," IEEE Access, vol. 6, pp. 52366-52 373, 2018.

[14] S. Gupta, S. Abielmona, and C. Caloz, "Microwave analog real-time spectrum analyzer (RTSA) based on the spectral-spatial decomposition property of leaky-wave structures," IEEE Trans. Microw. Theory Tech. vol. 57, no. 12, pp. 2989-2999, 2009.

[15] S. Gupta, C. Caloz, and S. Abielmona, "CRLH leaky-wave real-time spectrum analyzer (rtsa) with unrestricted time-frequency resolution," in 2008 IEEE MTT-S International Microwave Symposium Digest. IEEE, 2008, pp. 807-810.

[16] J. L. Gomez-Tornero, F. Quesada-Pereira, A. Alvarez-Melcon, G. Goussetis, A. R. Weily, and Y. J. Guo, "Frequency steerable two dimensional focusing using rectilinear leaky-wave lenses," IEEE Trans. Antennas Propagat., vol. 59, no. 2, pp. 407-415, 2010.

[17] M. Garcia-Vigueras, J. L. Gomez-Tornero, G. Goussetis, A. R. Weily, and Y. J. Guo, "1-D-leaky wave antenna employing parallel-plate waveguide loaded with PRS and HIS," IEEE Trans. Antennas Propagat., vol. 59, no. 10, pp. 3687-3694, 2011.

[18] A. J. Martinez-Ros, J. L. Gomez-Tornero, and G. Goussetis, "Planar leaky-wave antenna with flexible control of the complex propagation constant," IEEE Trans. Antennas Propagat., vol. 60, no. 3, pp. 16251630, 2011.

[19] D. J. King, M. K. Emara, and S. Gupta, "Millimeter-wave near-field spectrum analyzer based on integrated side-fire antennas," in 2019 13th European Conference on Antennas and Propagation (EuCAP). IEEE, 2019 , pp. $1-3$

[20] D. R. Jackson, C. Caloz, and T. Itoh, "Leaky-wave antennas," Proc. IEEE, vol. 100, no. 7, pp. 2194-2206, 2012.

[21] N. Yang, C. Caloz, and K. Wu, "Full-space scanning periodic phasereversal leaky-wave antenna," IEEE Trans. Microw. Theory Tech., vol. 58, no. 10, pp. 2619-2632, 2010.

[22] K. Sakakibara, J. Hirokawa, M. Ando, and N. Goto, "A linearlypolarized slotted waveguide array using reflection-cancelling slot pairs," IEICE Trans. Commun., vol. E77-B, no. 4, pp. 511-518, Apr. 1994.

[23] C. Caloz, T. Itoh, and A. Rennings, "CRLH metamaterial leaky-wave and resonant antennas," IEEE Antennas Propagat. Mag., vol. 50, no. 5, pp. 25-39, 2008

[24] S. Paulotto, P. Baccarelli, F. Frezza, and D. R. Jackson, "Full-wave modal dispersion analysis and broadside optimization for a class of microstrip CRLH leaky-wave antennas," IEEE Trans. Microw. Theory Tech., vol. 56, no. 12, pp. 2826-2837, 2008.

[25] R. E. Collin and F. J. Zucker, "Antenna theory,” 1969.

[26] D. M. Pozar, "Microwave engineering 3e," Tramsmission Lines and Waveguides, pp. 143-149, 2005.

[27] F. Arndt, I. Ahrens, U. Papziner, U. Wiechmann, and R. Wilkeit, "Optimized E-plane T-junction series power dividers," IEEE Trans. Microw. Theory Tech., vol. 35, no. 11, pp. 1052-1059, 1987.

[28] J. Hirokawa, K. Sakurai, M. Ando, and N. Goto, "An analysis of a waveguide T junction with an inductive post," IEEE Trans. Microw. Theory Tech., vol. 39, no. 3, pp. 563-566, 1991.

[29] A. Mallahzadeh and S. Mohammad-Ali-Nezhad, "Periodic collinearslotted leaky wave antenna with open stopband elimination," IEEE Trans. Antennas Propagat., vol. 63, no. 12, pp. 5512-5521, 2015.

[30] P. Baccarelli, P. Burghignoli, D. Comite, W. Fuscaldo, and A. Galli, "Open-stopband suppression via double asymmetric discontinuities in 1D periodic 2-D leaky-wave structures," IEEE Antennas Wirel. Propagat. Lett., vol. 18, no. 10, pp. 2066-2070, 2019.

[31] S. Otto and C. Caloz, "Importance of transversal and longitudina symmetry/asymmetry in the fundamental properties of periodic leakywave antennas," in 2013 IEEE Antennas and Propagation Society International Symposium (APSURSI). IEEE, 2013, pp. 240-241.

[32] S. Otto, A. Al-Bassam, A. Rennings, K. Solbach, and C. Caloz, "Transversal asymmetry in periodic leaky-wave antennas for Bloch impedance and radiation efficiency equalization through broadside," IEEE Trans. Antennas Propagat., vol. 62, no. 10, pp. 5037-5054, 2014.

[33] M. Guglielmi and D. Jackson, "Broadside radiation from periodic leakywave antennas," IEEE Trans. Antennas Propagat., vol. 41, no. 1, pp. 31-37, 1993

[34] L. Josefsson and P. Persson, Conformal array antenna theory and design. John Wiley \& Sons, 2006, vol. 29.

[35] C. A. Balanis, Antenna theory: analysis and design. John Wiley \& Sons, 2016.

[36] W. L. Stutzman and G. A. Thiele, Antenna theory and design. John Wiley \& Sons, 2012. 